\title{
Introduction: the Glycobiology of nervous system
}

\section{Sandro Sonnino ${ }^{1} \cdot$ Alessandro Prinetti $^{1}$}

Published online: 4 September 2018

(C) Springer Science+Business Media, LLC, part of Springer Nature 2018

\section{The Glycobiology of nervous system comes of age}

Glycoproteins, glycolipids and glycosaminoglycans are ubiquitous compounds in eukariotyc cells, and they play crucial roles in all aspects of cell and tissue biology.

It is a nonsense to prioritize the several biological processes crucial for human life, however probably any of us understands and agrees that without correct functions of the nervous system, also in the presence of a healthy situation of the remaining body, the life becomes poor if not useless. Glycobiology research associated with oncology and cardio-vascular diseases is thriving; however, it is clear from long time that glycans play major roles in the maintenance of the proper function of the nervous system. The complexity of this research area has considerably slowed down our comprehension of the regulation of glycans expression in the nervous system, but now we are fully appreciating that the deregulation of glycan expression is associated with devastating neurological diseases, and more and more Scientists are attracted to this field.

Several groups are today active in fields related to neuroglycobiology, and meetings in the field of Glycoscience include sessions or talks related to neurotopics. However, specific meetings dedicated to this theme were very scant in recent years.

With this in our minds, we decided to organize the 1st International Conference on the Glycobiology of

Sandro Sonnino

sandro.sonnino@unimi.it

1 Department of Medical Biotechnology and Translational Medicine, University of Milano, Milan, Italy
Nervous System. The meeting was held on September 2nd to September 5th, 2017, in jointly with the GlycoNeuro group of the Asian Pacific Society for Neurochemistry and the Korea University College of medicine, under the auspices of the International Society for Neurochemistry. We are grateful to the young scientists from the BK21PLUS graduate program (KU) and from the Department of Medical Biotechnology and Translational Medicine (UniMi) and to Mrs. So-young Yoon, who did a great job for the onsite organization. The meeting, with an attendance of over 80 , covered aspects related to the roles of glycoconjugates in both central and peripheral nervous system, providing a state-of-the art overview about the major advancements in this exciting field.

Some of these aspects have been now summarized in a few review papers that we present in a miniseries, with the precise scope of attracting into the neuroglycobiology world more Scientists and above all increasingly young Researchers.

We thank all the Authors that enthusiastically and in an exceptional way offered their contribute:

Neural functions of bisecting GlcNAc Yasuhiko Kizuka ${ }^{1}$ and Naoyuki Taniguchi ${ }^{2}$

${ }^{1}$ Gifu University and ${ }^{2}$ Osaka International Cancer Institute, Japan

Mental disorders and an acidic glycan- from the perspective of polysialic acid (PSA/polySia) and the synthesizing enzyme, ST8SIA2

Chihiro Sato and Masaya Hane

Nagoya University, Japan

Keeping it trim: roles of neuraminidases in CNS function. Alexey V. Pshezhetsky and Mila Ashmarina University of Montréal, Canada 
Heparan sulfate S-domains and extracellular sulfatases (Sulfs): their possible roles in protein aggregation diseases

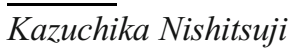

Tokushima University, Japan
Neuronal membrane dynamics as fine regulator of sphingolipid composition

Massimo Aureli, Maura Samarani, Nicoletta Loberto, Elena Chiricozzi, Laura Mauri, Sara Grassi, Domitilla Schiumarini, Alessandro Prinetti and Sandro Sonnino University of Milan, Italy

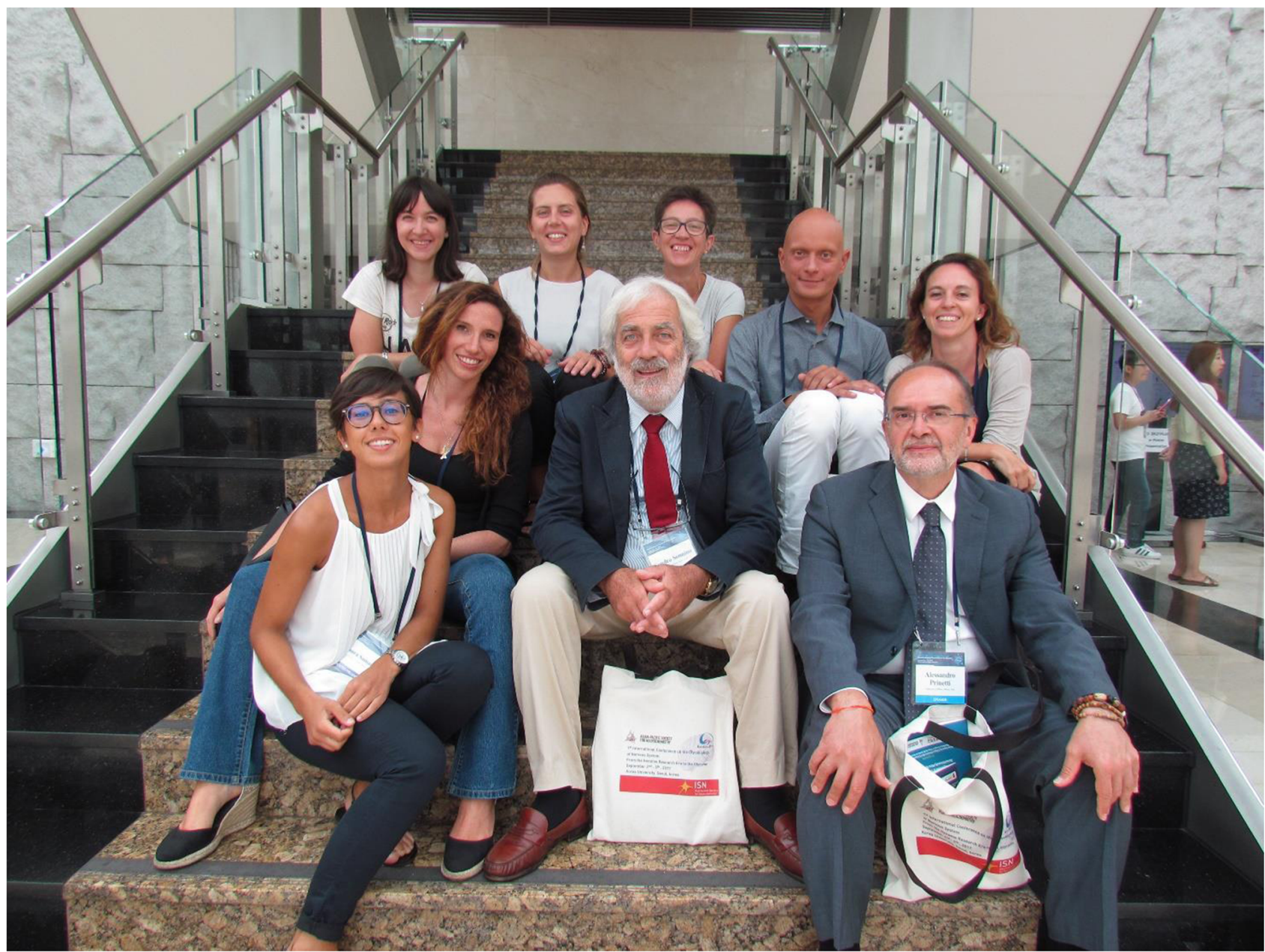

The group of the University of Milan that actively participated in the organization of the 1st International Conference on the Glycobiology of Nervous System.

From bottom left and clockwise: Maura Samarani, Domitilla Schiumarini, Sara Grassi, Elena Chiricozzi, Laura Mauri, Massimo Aureli, Nicoletta Loberto, Alessandro Prinetti, Sandro Sonnino. 